\title{
Magnetic Field Induced Redistribution of Exciton-Polariton Density on Confined Modes
}

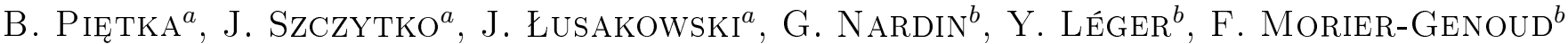 \\ AND B. Deveaud-PlÉdRAN ${ }^{b}$ \\ ${ }^{a}$ Institute of Experimental Physics, Faculty of Physics, University of Warsaw, Hoża 69, 00-681 Warsaw, Poland \\ ${ }^{b}$ Ecole Polytechnique Fédérale de Lausanne (EPFL), Lausanne, Switzerland
}

\begin{abstract}
The influence of magnetic field on confined exciton-polariton modes inside a semiconductor microcavity is discussed. The three-dimensional confinement for exciton-polaritons is achieved by the mesa structures confining the photonic part of polaritons. We observe a strong increase of the polariton emission intensity and we argue that this effect is due to the change of the oscillator strength of the excitonic component of polaritons and the change of the excitonic content in polariton state as the magnetic field increases.
\end{abstract}

PACS: 71.36.+c, 42.55.Sa, 71.35.-y, 71.35.Ji, 78.20.Ls

\section{Introduction}

Exciton-polaritons attracted worldwide interest due to their potential in polariton-based devices [1], possibility to create a Bose-Einstein condensate [2] or formation of a superfluid state with zero viscosity [3] with many spectacular properties.

Exciton-polaritons are the eigenmodes of a strongly coupled photonic and excitonic resonance inside the semiconductor microcavity [4]. The photonic part is due to the confinement in one dimension of the electromagnetic field by two distributed Bragg reflectors (DBRs) forming a microcavity. In the maximum of the cavity field a semiconductor quantum well is placed that provides the excitonic component to polaritons. Excitons confined inside the quantum well couple to the photonic modes, with the coupling strength given by the Rabi splitting. Excitonpolaritons can be considered as composite half-matter half-light quasi-particles. They have an advantage of having an extremely small effective mass of the order of $10^{-4}$ of the free electron mass which allows to confine them inside three-dimensional traps of the size of several $\mu \mathrm{m}$ [5-7] or to manipulate their confined states [8]. The micrometer size of the traps allows to use standard optics for imaging the confined state wave function, giving the full information about the real space amplitude and phase [9]. This property makes them a very promising system in contrast to semiconductor quantum dots which are orders of magnitude smaller and for which there is no easy optical access to image single state wave function.

In this study we use the external magnetic field to influence the excitonic component of polaritons. We demonstrate how the modification of the oscillator strength and excitonic diamagnetic shift influences the emission from the confined modes of polaritons. We concentrate here on the influence of magnetic field on the emission intensity changes from confined modes with comparison to the work of Ref. [10] where the emission energy shift was discussed in detail.

\section{Experiment}

The sample consists of a GaAs lambda microcavity sandwiched between two DBR AlAs / GaAs with one $8 \mathrm{~nm}$ thick InGaAs quantum well. The excitonic resonance is at $1.484 \mathrm{eV}(835.35 \mathrm{~nm})$ and the vacuum Rabi splitting is equal to $\Omega=3.5 \mathrm{meV}$. This is the same sample as described in detail in Ref. [11]. The polariton population is created non-resonantly and the laser is tuned to the first minimum of the Bragg reflectors. The sample is placed in a magnetic field up to $5 \mathrm{~T}$ in a Faraday configuration at the cold finger of an optical cryostat at the temperature of $5 \mathrm{~K}$. The photoluminescence $(\mathrm{PL})$ is collected through a microscope objective of a high numerical aperture $(\mathrm{NA}=0.5)$.

The traps for polaritons are made of mesas, a local circular extension of the cavity spacer thickness, which provides a lateral confinement for the photonic part of the polariton. The photonic modes are therefore confined in all three spatial dimensions which results in formation of discrete states. As the excitonic component is strongly coupled to each of the photonic modes, the discrete confined levels of the upper and lower polaritons are formed $[7,11]$.

\section{Results}

Figure 1 shows a spatially resolved PL spectrum from a mesa of $3 \mu \mathrm{m}$ in diameter in full spectral range. The two- 
-dimensional upper (2D UP) and lower (2D LP) polariton branches are visible in the region outside the mesa. The discrete polariton states are visible below 2D UP and $2 \mathrm{D}$ LP energies. In the following, we are investigating the effect of magnetic field effect on the lower polariton states confined in mesa of $3 \mu \mathrm{m}$ in diameter. We label these confined states with p1-p6 (see Figs. 1 and 2), starting from the mesa ground state. Their spatial profiles are those of a quasi-cylindrical confinement potential $[9,12]$.

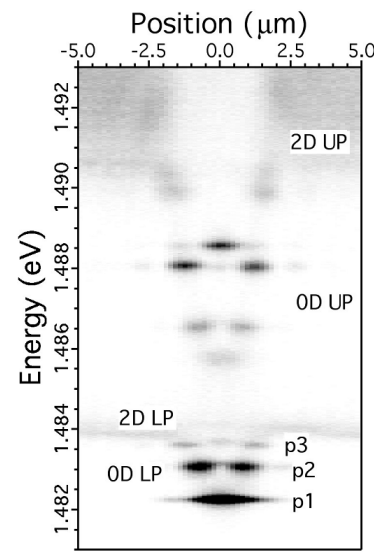

Fig. 1. The photoluminescence spectrum of a $3 \mu \mathrm{m}$ mesa excited nonresonantly. Position of $x=0 \mu \mathrm{m}$ corresponds to the centre of the mesa. The minimum value of intensity on the intensity scale is presented in white colour, the maximum in black, in arbitrary units. The colors are saturated to illustrate the emission from $2 \mathrm{D}$ states surrounding the mesa.

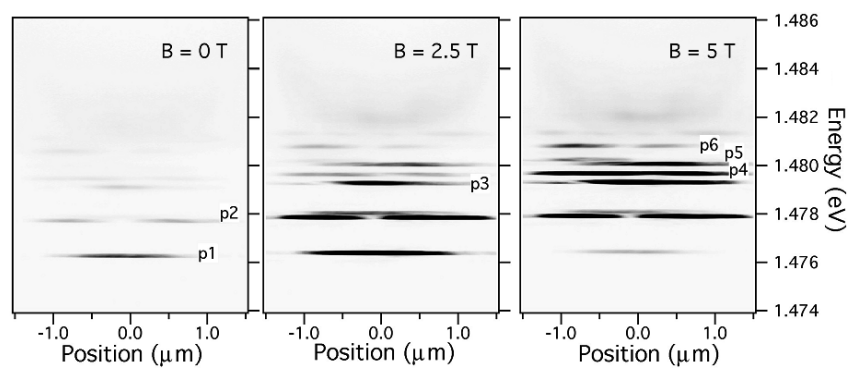

Fig. 2. The photoluminescence spectrum of a $3 \mu \mathrm{m}$ mesa in magnetic field of $0 \mathrm{~T}, 2.5 \mathrm{~T}$ and $5 \mathrm{~T}$ as marked in the images in the low polariton branch regime. The mesa is different one from that presented in Fig. 1, at more negative detuning. The minimum value of the intensity in the intensity scale is presented in white colour, the maximum in black, in arbitrary units. All images are in the same intensity scale.

Figure 2 illustrates the PL spectra in the magnetic field at $0 \mathrm{~T}, 2.5 \mathrm{~T}$, and $5 \mathrm{~T}$. We observe that the energy of the emission shifts to higher energy. This effect is expected as the magnetic field is acting mainly on the excitonic component of a polariton. The energy shift of a quantum well exciton can be described by a diamagnetic shift $\gamma$ in the first approximation of bound quasiparticle in the magnetic field $B$ by

$$
E_{x}\left(k_{\|}, B\right)=E_{x}(0)+\gamma B^{2},
$$

where $E_{x}(0)$ is the exciton emission energy at $0 \mathrm{~T}$. We neglect here the exciton Zeeman splitting as it is not resolved in our experiment. The energy of the lower $\left(E_{-}\right)$ and upper $\left(E_{+}\right)$polariton branches are given by

$$
E_{ \pm}\left(k_{\|}\right)=\frac{E_{\mathrm{c}}\left(k_{\|}\right)+E_{x}\left(k_{\|}\right)}{2} \pm \frac{1}{2} \sqrt{\delta^{2}\left(k_{\|}\right)+\hbar^{2} \Omega^{2}},
$$

where the $E_{x}$ and $E_{\mathrm{c}}$ are the excitonic and photonic resonances, respectively, the excitonic one given by Eq. (1).

This results in the evolution of the energy of the lower and upper polariton branches as illustrated in Fig. 3 for different exciton-photon detuning $\delta=E_{\mathrm{c}}\left(k_{\|}\right)-E_{x}\left(k_{\|}\right)$.

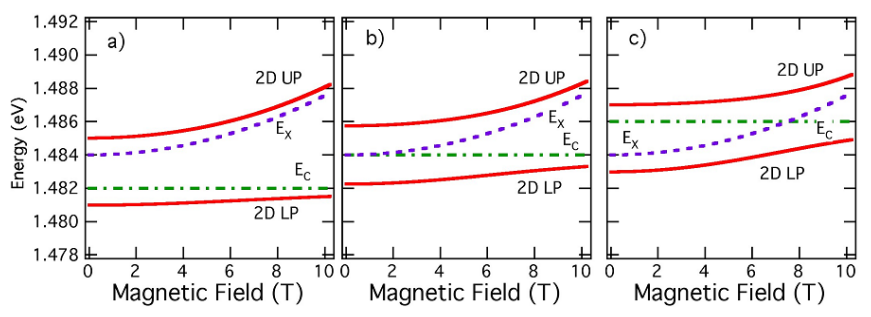

Fig. 3. The magnetic field evolution of the energy of upper and lower polariton at $k_{\|}=0$ according to Eq. (1) and Eq. (2) for (a) negative, (b) zero, and (c) positive detunings. The position of the bare cavity mode and exciton state are marked by dash-dotted and dash lines, respectively. The value of diamagnetic shift $\gamma=0.35 \mathrm{meV} / \mathrm{T}^{2}$ is taken from the $2 \mathrm{D}$ exciton shift observed in our experiment.

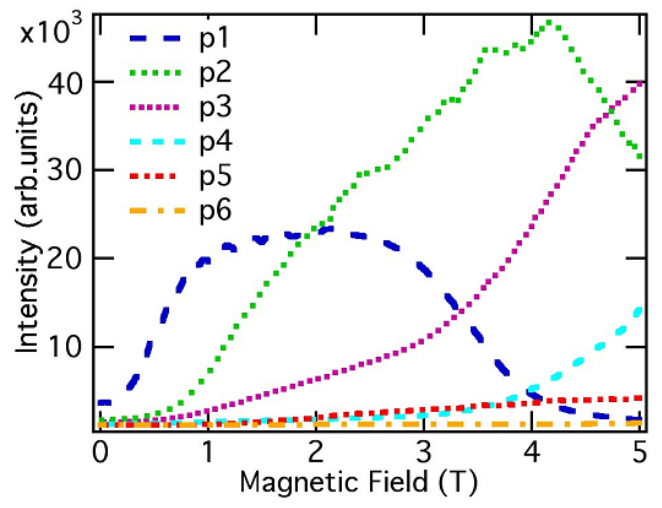

Fig. 4. The magnetic field evolution of the integrated intensity of the confined modes as marked in Fig. 2.

Apart from the energy shift, we observe a strong influence on the emission intensity from confined states, illustrated in Fig. 4. We observe that with increasing magnetic field the integrated intensity from a particular state increases and saturates. The saturation effect is observed at higher magnetic fields for higher energy states. The emission intensity on a given confined state depends strongly on the photonic fraction (the effective detuning for each of the state is different) and due to thermalisa- 
tion towards the lower energy state [13]. We believe that the magnetic field does not influence the thermalisation effect but modifies the excitonic and photonic content along the dispersion curve due to the energy shift. The excitonic oscillator strength is increased in magnetic field, which is responsible for the first increase of the emission intensity with $B$.

\section{Summary}

We observed a strong influence of the magnetic field on the optical transitions in the microcavity. First, it shrinks the electron and hole wave functions that lead to both exciton energy shift and modification of the exciton oscillator strength. This results in increase in the total emission intensity of polariton modes. Second, the magnetic field causes the quantization of the conduction and valence bands, which tunes the polariton emission energy. This effect can be used as effective tuning of the polariton emission energy as well as controlled detuning.

\section{Acknowledgments}

B.P., J.S., and J.Ł. acknowledge the support of the Ministry of Higher Education grant 2011/01/D/ST7/ 04088 .

\section{References}

[1] B. Deveaud-Plédran, Nature 453, 297 (2008).

[2] J. Kasprzak, M. Richard, S. Kundermann, A. Baas, P. Jeambrun, J.M.J. Keeling, F.M. Marchetti, M.H. Szymańska, R. André, J.L. Staehli, V. Savona, P.B. Littlewood, B. Deveaud, Le Si Dang, Nature 443, 409 (2006).

[3] A. Amo, J. Lefrère, S. Pigeon, C. Adrados, C. Ciuti, I. Carusotto, R. Houdré, E. Giacobino, A. Bramati, Nature Phys. 5, 805 (2009).
[4] C. Weisbuch, M. Nishioka, A. Ishikawa, Y. Arakawa, Phys. Rev. Lett. 69, 3314 (1992).

[5] J. Bloch, R. Planel, V. Thierry-Mieg, J.-M. Gérard, D. Barrier, J.-Y. Marzin, E. Costard, Superlatt. Microstruct. 22, 371 (1997).

[6] M. Obert, J. Renner, A. Forchel, G. Bacher, R. André, L.S. Dang, Appl. Phys. Lett. 84, 1435 (2004).

[7] R.I. Kaitouni, O. El Daif, A. Baas, M. Richard, T. Paraiso, P. Lugan, T. Guillet, F. Morier-Genoud, J.D. Ganière, J.L. Staehli, V. Savona, B. Deveaud, Phys. Rev. B 74, 155311 (2006).

[8] E. Wertz, L. Ferrier, D.D. Solnyshkov, R. Johne, D. Sanvitto, A. Lemaître, I. Sagnes, R. Grousson, A.V. Kavokin, P. Senellart, G. Malpuech, J. Bloch, Nature Phys. 6, 860 (2010).

[9] G. Nardin, R. Cerna, T.K. Paraïso, B. Pietka, Y. Léger, O. El Daif, F. Morier-Genoud, B. Deveaud-Plédran, Superlattices Microstruct. 47, 207 (2010).

[10] A. Rahimi-Iman, C. Schneider, J. Fischer, S. Holzinger, M. Amthor, S. Höfling, S. Reitzenstein, L. Worschech, M. Kamp, A. Forchel, Phys. Rev. B 84, 165325 (2011).

[11] O. El Daif, A. Baas, T. Guillet, J.-P. Brantut, R. Idrissi Kaitouni, J.L. Staehli, F. Morier-Genoud, B. Deveaud, Appl. Phys. Lett. 88, 061105 (2006).

[12] G. Nardin, Y. Léger, B. Pietka, F. Morier-Genoud, B. Deveaud-Plédran, Phys. Rev. B 82, 045304 (2010).

[13] T.K. Paraïso, D. Sarchi, G. Nardin, R. Cerna, Y. Léger, B. Pietka, M. Richard, O. El Daif, F. Morier-Genoud, V. Savona, B. Deveaud-Plédran, Phys. Rev. B 79, 045319 (2009). 